\title{
ANÁLISE DA ESTABILIDADE DA PRODUÇÃO DE LEITE NO MUNICÍPIO DE SANTO ÂNGELO - RS
}

\author{
Analysis of the milk production stability in \\ Santo Ângelo city - RS
}

Adriano Mendonça Souza', Gilvete Wolff Lírio² e Regiane klidzio² amsouza@smail.ufsm.br

' Universidade Federal de Santa Maria
${ }^{2}$ Universidade Regional Integrada

Resumo

O objetivo desta pesquisa é analisar a estabilidade da série produção de leite por meio de técnicas de controle estatístico de qualidade, de forma a propiciar às empresas que recebem o produto in natura uma estimativa dos períodos de maior e menor produção leiteira. Para cumprir o objetivo proposto, coletou-se uma amostra referente à produção de leite dos pequenos produtores do município de Santo Ângelo com uma amostra composta de cinquenta observações semanais medidas em litros no período de 20 de julho de 2009 a 12 de julho de 2010 . Inicialmente, analisou-se graficamente o comportamento da série e foi estimado o modelo SARIMA $(2,1,0)(1,0,1)_{7}$. Posteriormente, gráficos de controle foram aplicados na variável original e nos resíduos do modelo SARIMA, de modo a propiciar uma comparação entre os gráficos e compreender o efeito da autocorrelação quando se utilizam gráficos de controle. Ao comparar esses gráficos, constatou-se que a variável original, quando analisada por gráficos de controle, apresenta um maior número de pontos amostrais fora dos limites de controle do que quando 
aplicado o gráfico de controle aos resíduos do modelo matemático encontrado. A queda de produção leiteira apresenta uma característica sazonal, devido à escassez de alimentos no inverno e, no verão, pela escassez de água. Desse modo, salienta-se que os produtores devem criar métodos alternativos para estocar alimento para o gado e investir em rações alternativas. Já no verão, os criadores, além de se preocuparem com a alimentação, devem empreender um esforço extra para a manutenção de água para os animais e irrigação das lavouras para a pastagem natural. Além desses fatores, as condições sanitárias devem ser mantidas.

Palavras-chave: Séries Temporais. Modelos Box e Jenkins. Autocorrelação. Gráficos de Controle. Produção de Leite.

\section{Abstract}

The main objective of this research is to analyze the stability of the milk production series throughout the use of statistical process control techniques in a way to offer to the companies, which receive the product in natura an estimative of the periods of bigger and smaller milk production. To accomplish the main purpose, a sample was collated of some small producers in Santo Ângelo city. The sample was composed by fifty weeks from July $20^{\text {th }}, 2009$ toJuly $12^{\text {th }}, 2010$. Firstly, the series behavior was analyzed graphically and aftera $\operatorname{SARIMA}(2,1,0)(1,0,1)_{7}$ model was found.A control charts was established in the original variable and in the residues data from the SARIMA model, as a way to understand the autocorrelation effect in the control chart. Making a comparison among these control charts is possible to understand the autocorrelation effect present in the original data. Based on the original charts it is possible to verify that the series has a seasonal period represented by summer and winter months. Comparing the control charts, the original data presented more out-of-control sample points besides control limits than the residual series of the math model. It is necessary to emphasize that the producers should create alternative methods in order to maintain a minimum stock of cattle feed. In the summer is necessary to maintain an enough level of water to the cattle and to irrigate the pasture. Besides these factors the sanitary conditions must be maintained.

Keywords: Temporal Series. Box and Jenkins Models. Autocorrelation, Control Charts. Milk Production. 


\section{Introdução}

A produção de leite é uma das mais importantes atividades do setor agroindustrial brasileiro. Entre 1990 e 2000 , a produção nacional de leite cresceu $37 \%$. No entanto, esse aumento para os pequenos (até 50 litros/dia) e para os médios produtores (até 200 litros/dia) não foi representativo.

Segundo dados do censo agropecuário, realizado em 2006 pelo IBGE, o município de Santo Ângelo possui 1.902 estabelecimentos agropecuários, dos quais 1.611 são considerados do tipo familiar e 291 do tipo não familiar. Estima-se que em torno de 600 famílias santo-angelenses trabalhem na atividade leiteira.

A partir de 1996, o Governo Federal, sob o pretexto de melhorar a qualidade do leite, discutiu novas regras para a produção, privilegiando o grande em detrimento do pequeno produtor familiar, o qual reduziu ainda mais a sua produção. E, nesta cadeia produtiva, só se mantêm no mercado produtores que consigam reduzir os seus custos e aumentar o volume de leite produzido (LÍRIO et al., 2003), obtendo um ganho em escala.

A maior parte do leite é recolhida nas propriedades rurais por caminhões equipados com tanque resfriador, sendo transportada para empresas como: Bom Gosto, Nestlé, Brasil Foods, CCGL, entre outras. As condições favoráveis do clima e do solo na região proporcionam um aumento significativo da produção leiteira, fazendo com que essas empresas aumentem o potencial de produção de laticíneos da região.

Diante dessa perspectiva, o objetivo desta pesquisa é analisar e verificar a estabilidade da série de leite produzido em Santo Ângelo por meio da utilização de técnicas de controle estatístico de qualidade. Além disso, a pesquisa pretende propiciar às empresas que recebem o leite uma estimativa dos períodos de maior e menor produção.

\section{Metodologia}

Em consonância com o objetivo deste projeto, recorre-se à aplicação dos gráficos de controle para medidas individuais e amplitude móvel, com o intuito de identificar algum padrão atípico. Sabe-se que, para a aplicação dos gráficos de controle (GC), alguns pressupostos devem ser considerados, tais como independência e não autocorrelação. Também é necessário que as observações sejam normalmente distribuídas. 
Dessa forma, busca-se verificar graficamente o comportamento da série em estudo, com o propósito de averiguar a estacionariedade, as características sazonais e a presença de autocorrelação nos dados.

Se a autocorrelação estiver presente, a aplicação de gráficos de controle torna-se inadequada, pois viola os princípios da independência e não autocorrelação. Logo, é necessário tratar a autocorrelação por meio da modelagem ARIMA (Auto Regressive Integrate Moving Average) e/ou SARIMA (Sesonal Auto Regressive Integrate Moving Average), a qual produzirá resíduos livres de autocorrelação.

Estando os resíduos livres de autocorrelação, a próxima etapa é a aplicação de gráficos de controle $\bar{x}$ e $\overline{M R}$ para medidas individuais, os quais serão aplicados aos dados originais e aos resíduos oriundos do modelo ARIMA e/ou SARIMA estimado. Esse procedimento será adotado apenas para mostrar que a autocorrelação presente nos dados pode levar a conclusões errôneas.

Os dados foram coletados na Associação Rio-grandense de Empreendimentos de Assistência Técnica e Extensão Rural - EMATER- RS, no período de 20 de julho de 2009 a 12 de julho de 2010 , com observações semanais, medidas em litros, totalizando 50 observações.

Os softwares utilizados para analisar os dados foram: Statistica 7.0, EViews 7.0 e planilha eletrônica.

\section{Revisão de literatura}

Nesta etapa, apresentam-se as duas técnicas que serão úteis para o desenvolvimento do artigo.

\subsection{Metodologia de Box e Jenkins}

O método de Box e Jenkins, (1970) consiste na busca de um modelo ARIMA que represente o processo estocástico gerador da série temporal. Para a modelagem de séries temporais, é importante ter séries estacionárias, de forma que as estimativas dos parâmetros sejam estáveis ao longo do período de análise. Como grande parte das séries temporais são naturalmente não estacionárias, é necessário aplicar transformações para torná-las estacionárias. Geralmente, utilizam-se uma ou duas diferenças, que são suficientes para torná-la estacionária(BUENO, 2008).

Os modelos ARIMA são eficientes para capturar o comportamento da série de dados e o comportamento da série pode ser representado 
por meio dos filtros AR (parâmetros autorregressivos - $p$ ) e MA (parâmetros de médias móveis - q). O número de diferenciações necessárias para tornar a série estacionária é dado pelo fator $d$. Dessa forma, tem-se o modelo geral ARIMA $(p, d, q)$.

Segundo Morettin (2006), os modelos ARIMA são responsáveis por capturar e modelar processos de memória curta, pois a FAC decresce rapidamente para zero. Caso o processo não tenha essa característica, outros modelos devem ser empregados para estimar processos de memória longa.

Seja $Z_{t}$ de modo que $\left\{x_{t}, t \in Z\right\}$ segue um processo ARIMA $(p, d, q)$ com $d$ diferenças $\Delta^{d} x_{t}$ se:

$$
\phi(B) \Delta^{d} x_{t}=\theta(B) e_{t}
$$

em que $B$ é operador retroativo ou operador de defasagens, $d$ é um inteiro positivo e representa o número de diferenças para tornar a série estacionária, também denominado de ordem de integração; $\phi$ e $\theta$ são os parâmetros dos processos autorregressivos e de médias móveis de ordem $p$ e $q$, respectivamente, $e_{t}$ é um processo ruído branco com média zero e variância constante, $e_{t} \sim R B\left(0, \sigma^{2}\right)$.

Os modelos ARIMA exploram a autocorrelação entre os valores da série em instantes sucessivos. Entretanto, quando os dados são observados em períodos inferiores a um ano, a série também pode apresentar autocorrelação para períodos fixos durante o ano, a qual pode ser caracterizada como sazonalidade e geralmente coincide com uma determinada repetição dentro do ano, como estações de chuvas, secas e temperaturas elevadas, entre outros fatores. Os períodos de sazonalidade são representados por $s$ e apresentam autocorrelação nos intervalos sazonais, sendo representados pelos modelos SARIMA (p, d, q)(P, D, Q) (WERNER e RIBEIRO, 2003).

A construção de modelos ARIMA segue um ciclo iterativo da metodologia de Box e Jenkins, composto por quatro etapas: $a$ ) identificação; b) estimação; c) verificação do diagnóstico e d) previsão (GUJARATI, 2000).

Após a identificação do modelo matemático que seja uma representação adequada do mecanismo gerador da série, a estimação dos parâmetros desse modelo é efetuada (HILL, GRIFFITHS e JUDGE, 1999). 
Posteriormente, realiza-se uma validação dos modelos por meio do critério de informação de Akaike (AIC), caracterizado por ser um dos critérios penalizadores, pois leva em consideração o número de parâmetros $n$ para representar a série para os modelos de previsão.

$$
A I C=\mathrm{T} \ln (\mathrm{SOR})+2 n
$$

Em que: T é o tamanho da amostra; SQR é a soma dos quadrados dos resíduos; e $n$ é o número de parâmetros.

\subsection{Gráficos de controle}

Os gráficos de controle auxiliam na melhoria contínua da qualidade do produto. Eles podem ser usados para reduzir não conformidades em tempo real, bem como mostrar, quando necessário, a necessidade da existência de ações no processo que está sendo monitorado.

O objetivo principal da aplicação dos gráficos de controle é verificar se o processo em análise é estatisticamente estável, indicando quando é necessário atuar sobre ele. O aprimoramento contínuo do processo se dá mediante a redução de sua variabilidade (RYAN, 1989); (MONTGOMERY, 1997).

Em geral, um gráfico de controle pode indicar uma condição de instabilidade, sinalizando quando um ou mais pontos estão além dos limites de controle, ou quando exibem algum padrão de comportamento não aleatório. Segundo Klidzio e Souza (2009), um processo pode ser considerado fora de controle, mesmo quando todos os pontos amostrais estão dentro dos limites de controle. Se ocorrer um padrão de variação anormal no processo e os pontos amostrais forem sinalizados pelos testes de sequência ou runs tests, ${ }^{1} q u e$ revelam padrões não aleatórios, o processo também será considerado fora de controle.

Os gráficos de controle para medidas individuais e amplitude móvel são conhecidos por gráficos de $\bar{x}$ e $\overline{M R}$ e possuem a habilidade para detectar grandes deslocamentos na média do processo.

A média entre as amostras, a amplitude móvel e a média da amplitude móvel são definidas respectivamente por:

\footnotetext{
' Runs tests, também conhecidos por testes de sequência, servem para identificar os pontos amostrais fora de controle, em busca de padrões não aleatórios.
} 


$$
\bar{x}=\frac{x_{1}+x_{2}+\cdots+x_{n}}{n}
$$

em que $x_{1}, x_{2}, \ldots, x_{n}$ é uma amostra de tamanho $n$, e $\bar{x}$ é a média dessa amostra.

$$
M R_{i}=\left|x_{i}-x_{i-1}\right|
$$

em que: $M R_{i}$ é a amplitude móvel de duas observações consecutivas como base para estimar a variabilidade do processo.

$$
\overline{M R}=\frac{M R_{1}+M R_{2}+\cdots+M R_{n}}{n}
$$

em que $\overline{M R}$ é a média das amplitudes móveis.

Para o gráfico de medidas individuais $(\bar{x})$, os limites situados a três desvios-padrão da média são dados por:

$$
\begin{gathered}
L S C=\bar{x}+3 \frac{\overline{M R}}{d_{2}} \\
L C=\bar{x} \\
L I C=\bar{x}-3 \frac{\overline{M R}}{d_{2}}
\end{gathered}
$$

Para o gráfico de amplitude móvel $(\overline{M R})$, os limites de controle são dados pelas expressões:

$$
\begin{gathered}
L S C=D_{4} \overline{M R} \\
L C=\overline{M R} \\
L I C=D_{3} \overline{M R}
\end{gathered}
$$

As constantes $d_{2}, D_{4}$ e $D_{3}$ encontram-se tabuladas em Montgomery (2004).

Para a análise da estabilidade do processo produtivo, deve-se ter uma atenção especial às variações incomuns ou sistemáticas, visto que as 
variações aleatórias dificilmente fazem com que o processo esteja fora de controle. Assim, o processo será considerado sob controle nas seguintes condições:

1)todos os pontos estão compreendidos nos limites de controle superior e inferior e não seguem nenhuma variação sistemática conforme mostra a Figura 1;

2) o gráfico apresenta, aproximadamente, o mesmo número de pontos acima e abaixo da linha central;

3) a maioria dos pontos (cerca de dois terços) situa-se próxima à linha central, conforme apresenta a Figura 2;

4) poucos pontos situam-se próximos aos limites de controle.

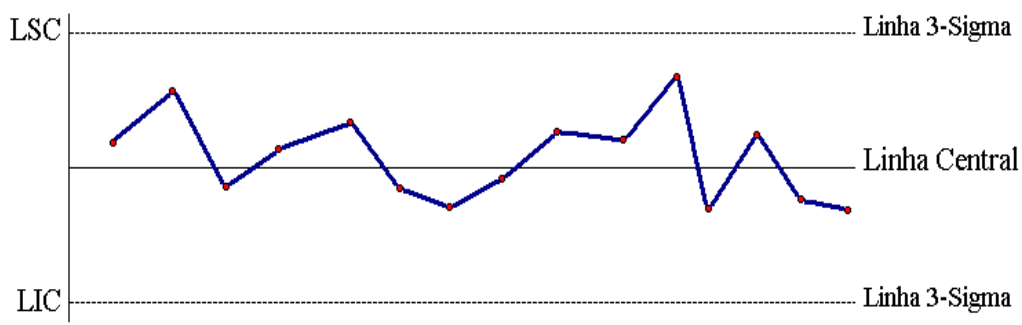

Figurar. Processo considerado sob controle estatístico.

Na Figura 2, são mostrados os intervalos dos limites de controle, divididos em seis zonas, com o valor de $k$ variando de 1 a 3 . Essas zonas entre os limites são descritos como A, B e C, e nelas verifica-se a distribuição dos pontos. Esse procedimento é denominado "runs tests".

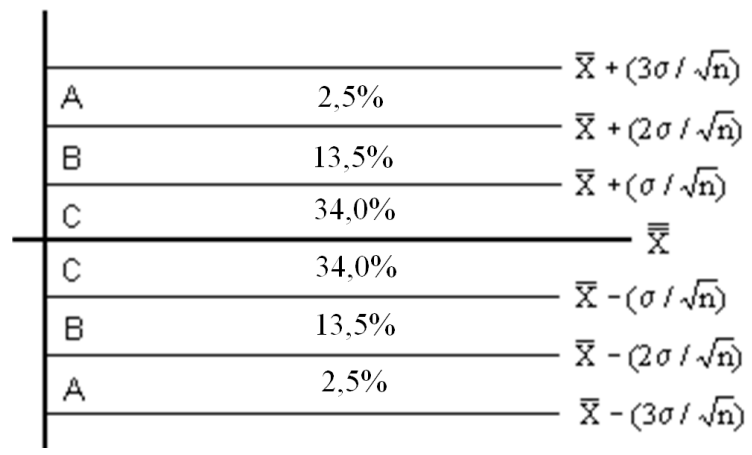

Figura2. Gráfico de Controle de Shewhart com os limites de controle. Fonte: Adaptada de Montgomery e Runger (2003). 
Sabe-se que apenas a observância de um ponto fora dos limites de controle não é suficiente para informar sob a estabilidade do processo. Por essa razão, Siqueira (1997) mostrou oito situações que podem ocorrer em gráficos de controle, baseadas no comportamento das variáveis que podem revelar alguma instabilidade no processo, sugerindo-se, assim, que algum procedimento alternativo deva ser executado, pois o processo corre o risco de sair de controle.

Segundo a análise do gráfico da Figura 2, subdividido em zonas, quando ocorrer uma das oito configurações descritas na Figura 3, o processo será considerado fora de controle.

\begin{tabular}{|c|c|}
\hline $\begin{array}{l}1^{\text {a }} \text { configuração: } \\
\text { Um ponto além da zona A, isto } \\
\text { é, acima do limite superior de con- } \\
\text { trole ou abaixo do limite inferior } \\
\text { de controle. }\end{array}$ & $\overline{y-1}$ \\
\hline $\begin{array}{l}2^{\mathrm{a}} \text { configuração: } \\
\text { Nove pontos sucessivos de um } \\
\text { mesmo lado do valor central, ou } \\
\text { seja, todos acima ou abaixo da li- } \\
\text { nha média. }\end{array}$ & \begin{tabular}{|l|}
$A$ \\
$B$ \\
$C$ \\
$C$ \\
$B$ \\
$A$
\end{tabular} \\
\hline $\begin{array}{l}\quad 3^{\mathrm{a}} \text { configuração: } \\
\text { Seis pontos sucessivos aumen- } \\
\text { tando ou diminuindo constante- } \\
\text { mente. }\end{array}$ & 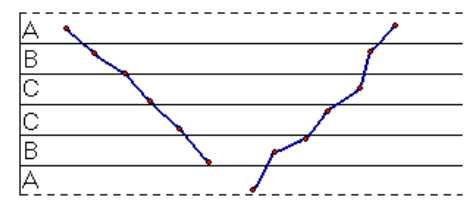 \\
\hline $\begin{array}{l}4^{\mathrm{a}} \text { configuração: } \\
\text { Quatorze pontos sucessivos al- } \\
\text { ternando-se para cima e para baixo. }\end{array}$ & $\mid \begin{array}{l}A \\
B \\
C\end{array}$ \\
\hline
\end{tabular}




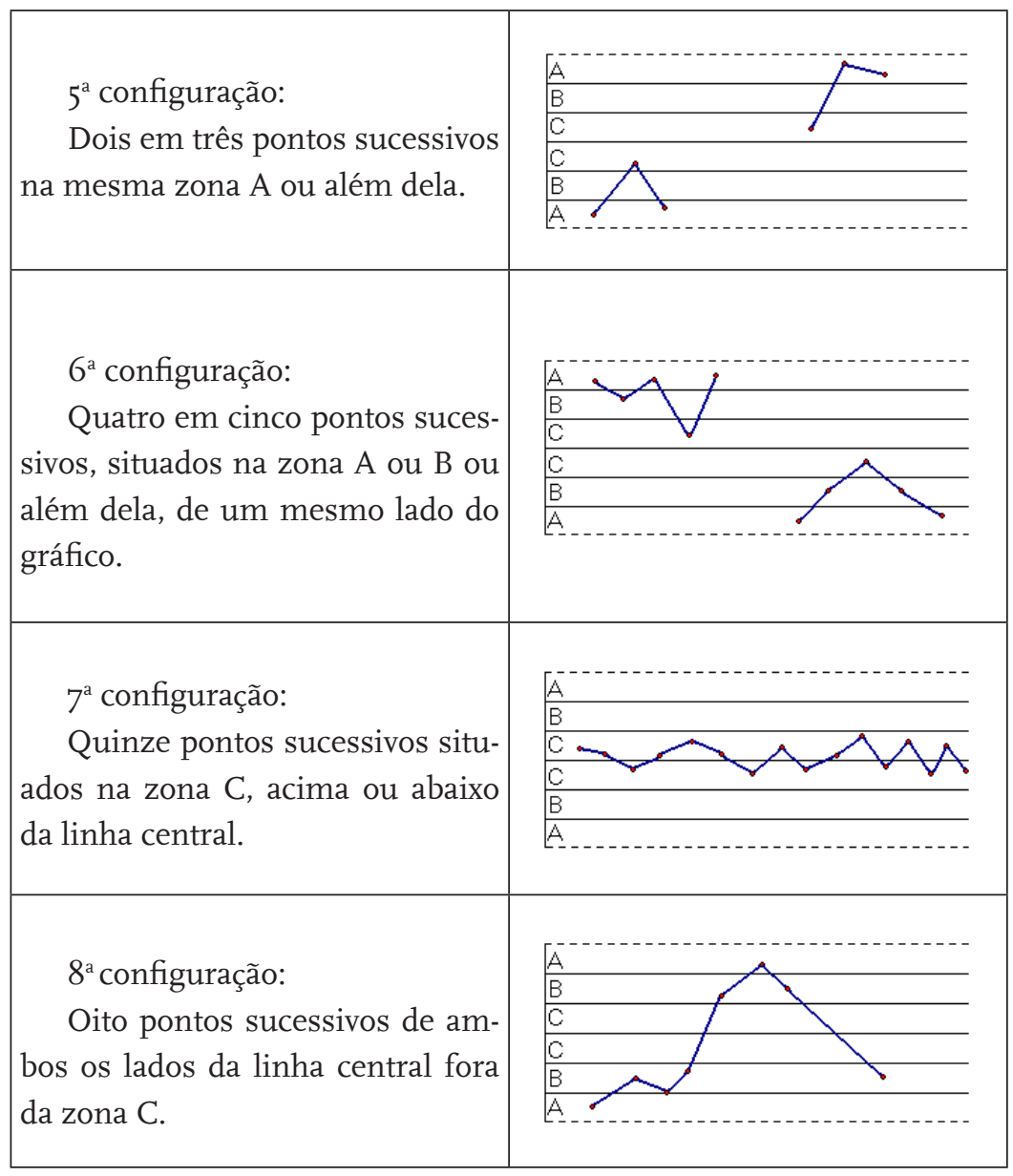

Figura 3. Configurações que indicam um processo fora de controle. Fonte: Adaptada de Siqueira (1997).

\section{Análise e discussão dos resultados}

Para a análise da série produção de leite em litros, inicialmente, faz-se uma inspeção visual, conforme a Figura 4. Constata-se um movimento com características sazonais, pois no verão percebe-se um aumento na produção de leite, atingindo um valor máximo de 40.000 litros e, nos meses de inverno, um declínio, registrando produção mínima de 28.000 litros. 


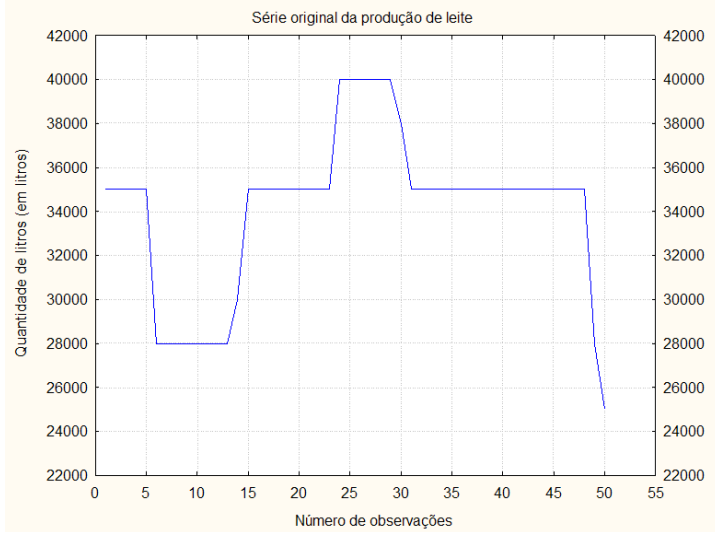

Figura 4. Série original da produção de leite semanal no período em estudo.

A componente sazonal pode ser entendida como a parte da série temporal que apresenta um movimento típico de aumento e/ou diminuição de produção ao longo do tempo, que pode ser associada aos períodos de inverno e verão. Também se verifica, pela inspeção visual, que a série parece ser não estacionária, em que a primeira investigação se faz pelas funções de autocorrelação (FAC) e de autocorrelação parcial (FACP), apresentada na Figura 5, a qual mostra um decaimento não muito rápido para zero, o que é um forte indício de não estacionariedade. Além disso, é possível notar na FACP uma oscilação das autocorrelações parciais, sinalizando algum tipo de sazonalidade. Dessa forma, devem-se tomar diferenças para estacionarizar a série e utilizar um modelo que capte a sazonalidade da série.

\begin{tabular}{|c|c|c|c|c|c|c|c|c|}
\hline \multicolumn{2}{|c|}{ FAC } & \multicolumn{2}{|c|}{ FACP } & & $A C$ & PAC & Q-Stat & Prob \\
\hline 1 & $\square$ & 1 & 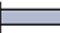 & 1 & 0.708 & 0.708 & 26.626 & 0.000 \\
\hline 1 & $\square$ & 1 & 曰I & 2 & 0.598 & 0.193 & 45.989 & 0.000 \\
\hline 1 & $\square$ & 1 & 1 & 3 & 0.497 & 0.038 & 59.633 & 0.000 \\
\hline 1 & $\square$ & 1 & | & 4 & 0.507 & 0.203 & 74.151 & 0.000 \\
\hline 1 & $\square$ & $\square$ & 1 & 5 & 0.322 & -0.275 & 80.131 & 0.000 \\
\hline 1 & إ & 1맘 & 1 & 6 & 0.167 & -0.205 & 81.784 & 0.000 \\
\hline 1 & $p 1$ & 10 & 1 & 7 & 0.045 & -0.095 & 81.907 & 0.000 \\
\hline 1 & 1 & 1 & p & 8 & 0.052 & 0.088 & 82.075 & 0.000 \\
\hline 1 & 1 & 1 & פי & 9 & 0.059 & 0.209 & 82.295 & 0.000 \\
\hline 1 & 1 & 1 & p 1 & 10 & 0.029 & 0.080 & 82.351 & 0.000 \\
\hline 1 & 1 & 1 & 1 & 11 & -0.015 & -0.027 & 82.366 & 0.000 \\
\hline 1 & 1 & 10 & 1 & 12 & -0.015 & -0.104 & 82.381 & 0.000 \\
\hline 1 & 1 & 1 & 1 & 13 & 0.030 & -0.051 & 82.444 & 0.000 \\
\hline 1 & ? 1 & 1 & 1 & 14 & 0.058 & 0.029 & 82.684 & 0.000 \\
\hline I & 1 & 1 & 1 & 15 & 0.045 & 0.051 & 82.832 & 0.000 \\
\hline
\end{tabular}

Figura 5. FAC e FACP da série produção de leite semanal. 
Após tornar a série estacionária por meio de diferenças, o próximo passo é identificar o mecanismo gerador da série, de modo que os efeitos da autocorrelação sejam captados por meio da escolha de um modelo matemático representativo para a série. No entanto, para que os resíduos oriundos desse modelo possam ser utilizados na aplicação dos gráficos de controle, eles devem atender aos pressupostos de independência, ser não autocorrelacionados e seguir uma distribuição Normal.

Dentre os vários modelos investigados, foram encontrados cinco tipos que apresentaram resíduos com características de processo de ruído branco. Esses modelos são denominados de concorrentes, pois todos atenderam a condição de ser ruído branco. Todavia, como é necessário optar por um modelo específico, recorreu-se ao critério AIC, que penaliza o modelo, considerando o número de parâmetros presentes utilizados na estimação, sendo, então, um critério de escolha entre os modelos concorrentes.

Na Tabela 1, encontram-se os modelos mais apropriados para representar a produção de leite, seus respectivos parâmetros e os valores de AIC.

Tabela 1. Modelos concorrentes para a série produção de leite semanal.

\begin{tabular}{|c|c|c|}
\hline Modelos & Parâmetros & AIC \\
\hline \multirow{4}{*}{$\operatorname{ARIMA}(3,1,1)$} & $p(1)=-0,3270$ & 18,03 \\
\hline & $\mathrm{p}(2)=0,0936$ & \\
\hline & $p(3)=-0,0098$ & \\
\hline & $q(1)=-0,5544$ & \\
\hline \multirow{4}{*}{$\operatorname{SARIMA}(2,1,0)(1,0,1)_{7}$} & $p(1)=0,2112$ & 17,93 \\
\hline & $p(2)=-0,0270$ & \\
\hline & $\operatorname{Ps}(1)=-0,88_{51}$ & \\
\hline & $Q s(1)=-0,8289$ & \\
\hline \multirow{6}{*}{$\operatorname{SARIMA}(2,1,0)(1,0,3)_{7}$} & $p(1)=0,2174$ & 17,98 \\
\hline & $p(2)=-0,0332$ & \\
\hline & $\operatorname{Ps}(1)=-0,0900$ & \\
\hline & $\mathrm{Qs}(1)=0,0025$ & \\
\hline & $\mathrm{Qs}(2)=-0,0086$ & \\
\hline & $Q s(3)=-0,0062$ & \\
\hline \multirow{5}{*}{$\operatorname{SARIMA}(3,1,0)(2,0,0)_{7}$} & $p(1)=-0,3270$ & 17,83 \\
\hline & $p(2)=-0,0090$ & \\
\hline & $p(3)=0,0664$ & \\
\hline & $\operatorname{Ps}(1)=-0,0271$ & \\
\hline & $\operatorname{Ps}(2)=0,0102$ & \\
\hline
\end{tabular}


É importante frisar que todos os parâmetros dos modelos apresentados na Tabela 1 são significativos. No entanto, o melhor modelo para representar a série produção de leite é o $\operatorname{SARIMA}(2,1,0)(1,0,1)_{7}$ por apresentar menor número de parâmetros.

Apresentam-se, na Tabela 2, os intervalos de confiança das estimativas dos parâmetros para o modelo matemático encontrado.

Tabela 2. Intervalos de confiança das estimativas dos parâmetros para o modelo SARIMA $(2,1,0)(1,0,1)_{7}$.

\begin{tabular}{cccc}
\hline Parâmetros & p-valor & $\begin{array}{c}\text { Limite inferior } \\
90 \%\end{array}$ & $\begin{array}{c}\text { Limite superior } \\
90 \%\end{array}$ \\
\hline $\mathrm{p}(1)=0,2112$ & 0,216712 & $-0,12832$ & 0,550754 \\
$\mathrm{p}(2)=-0,0269$ & 0,882342 & $-0,39210$ & 0,338131 \\
$\operatorname{PS}(1)=-0,8851$ & 0,530391 & $-3,70457$ & 1,934324 \\
$\mathrm{Qs}(1)=-0,8288$ & 0,584863 & $-3,86276$ & 2,205024 \\
\hline
\end{tabular}

Nas Figuras 6 e 7, apresentam-se a FAC e a FACP dos resíduos do modelo selecionado, as quais não evidenciam uma estrutura de autocorrelação, satisfazendo uma das condições para a aplicação dos gráficos de controle.

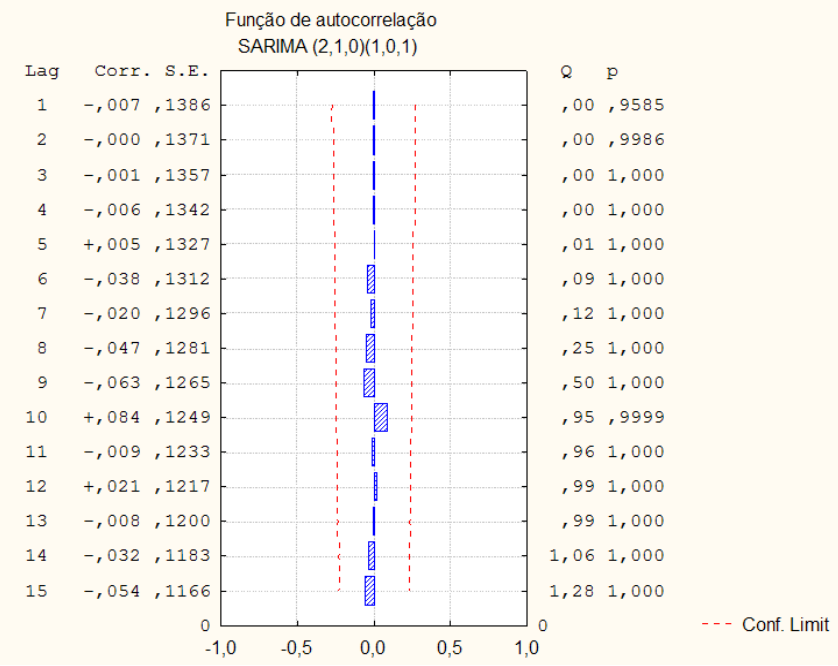

Figura 6. FAC dos resíduos do modelo ARIMA. 


\section{ciênciaenatura UFSM, 34(2)}

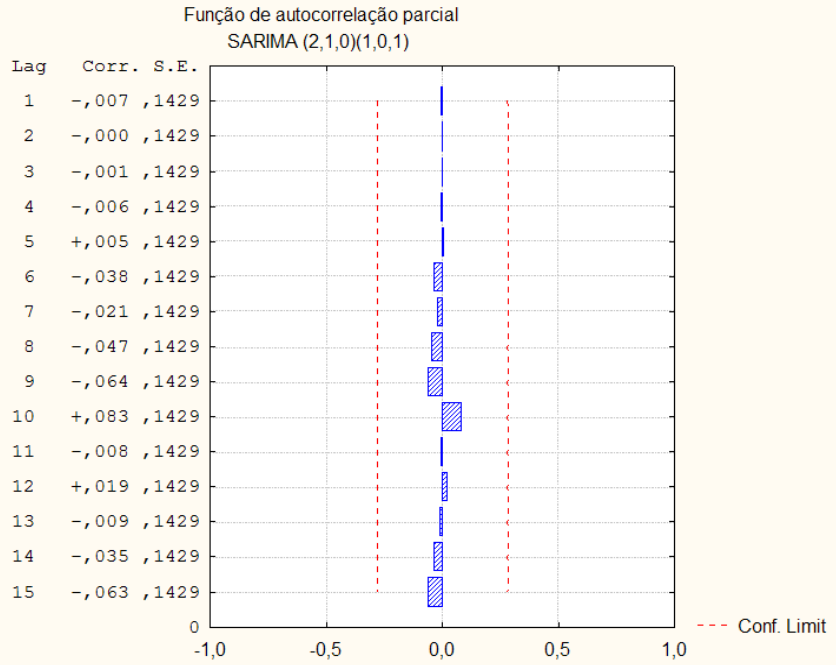

Figura 7. FACP dos resíduos do modelo ARIMA.

Com o objetivo de verificar visualmente a existência do efeito heteroscedástico nos dados, aplicam-se a FAC e a FACP aos resíduos quadráticos do modelo matemático encontrado, conforme mostram as Figuras 8 e 9, as quais mostram que não há sinais de volatilidade condicional.

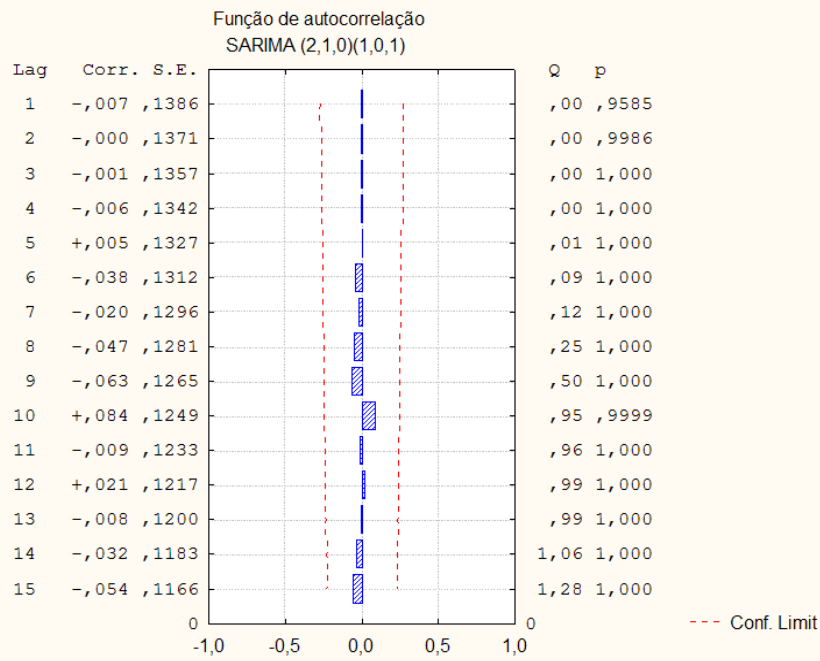

Figura 8. FAC dos resíduos quadráticos do modelo ARIMA. 


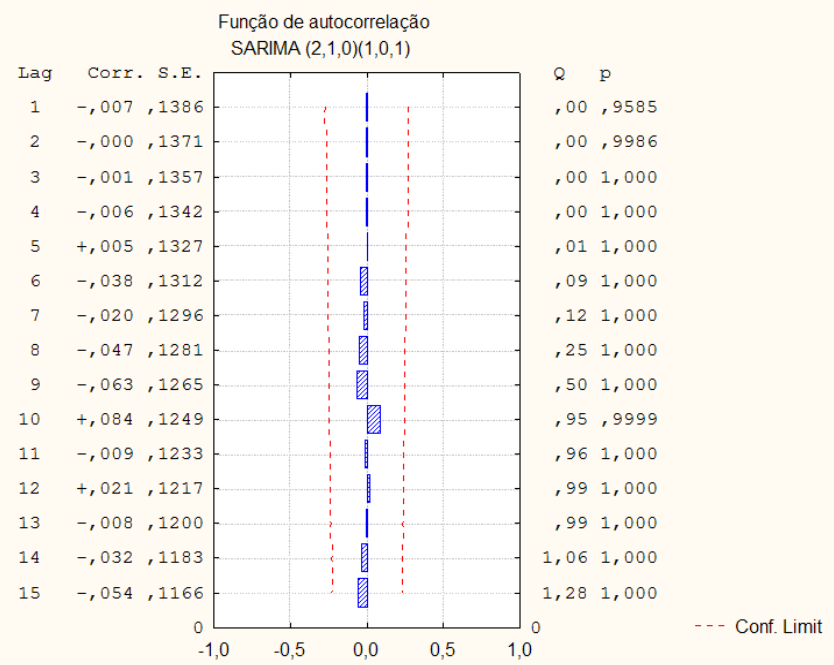

Figura 9. FACP dos resíduos quadráticos do modelo ARIMA.

Além da inspeção visual, para detectar estatisticamente a presença de volatilidade condicional, aplicou-se o teste de hipótese ARCH-LM nos resíduos quadráticos do modelo SARIMA $(2,1,0)(1,0,1)_{7}$. Encontra-se, na Tabela 3, o resultado desse teste, em que foram testadas as seguintes hipóteses:

$H_{0}$ : Não existem efeitos ARCH (homocedástica),

$H_{1}$ : Existem efeitos ARCH (não homocedástica).

Tabela3. Resultados das estatísticas F e ARCH-LM.

\begin{tabular}{ccccc}
\hline Lags & Estatística F & $p$-valor & $\begin{array}{c}\text { Estatística } \\
\text { ARCH-LM }\end{array}$ & $p$-valor (ARCH-LM) \\
\hline $\operatorname{Lag} 1$ & 0,157 & 0,694 & 0,164 & 0,686 \\
$\operatorname{Lag} 5$ & 0,374 & 0,863 & 2,078 & 0,839 \\
$\operatorname{Lag} 10$ & 4,699 & 0,001 & 23,499 & 0,009 \\
$\operatorname{Lag} 15$ & 2,458 & 0,046 & 22,035 & 0,107 \\
$\operatorname{Lag} 20$ & 1,200 & 0,459 & 21,516 & 0,367 \\
\hline
\end{tabular}

A partir dos resultados encontrados para as diferentes defasagens (lags), verifica-se que, na maioria delas, as estatísticas F e ARCH-LM não 
foram significativas, com exceção do lag 15. Assim, aceita-se a hipótese nula, implicando que não existem efeitos ARCH nos resíduos quadráticos do modelo SARIMA $(2,1,0)(1,0,1)_{7}$.

Após ter a certificação de que os resíduos não apresentaram sinais de dependência, dá-se início à aplicação dos gráficos de controle para medidas individuais e amplitude móvel.

Para evidenciar o efeito de autocorrelação, elaboram-se gráficos de controle nas variáveis originais, as quais apresentam autocorrelação, conforme mostram as Figuras 10 e 11. Nos gráficos de controle $\bar{x}$ e $\overline{M R}$, foram adicionados os runs tests, os quais podem revelar a existência de algum tipo de padrão nos dados.

X: $34100,(34100$,$) ; Sigma: 614,93 (614,93); n: 1,$

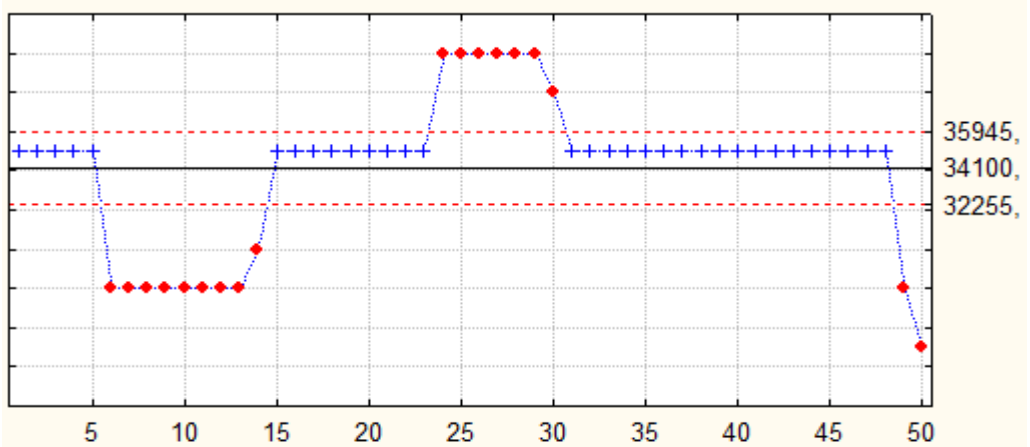

Figura 10. Gráfico $\bar{x}$ da série produção de leite semanal original.

MR: 693,88 (693,88); Sigma: 524,23 (524,23); n: 1,

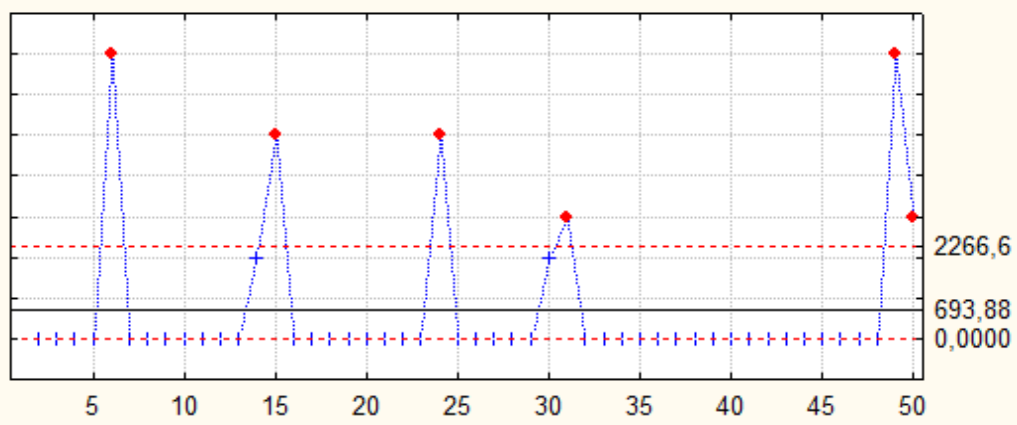

Figura 11. Gráfico $\overline{M R}$ da série produção de leite semanal original. 
Pela análise das Figuras 10 e 11, demonstra-se, pelo gráfico $\bar{x}$,que existem vários pontos fora dos limites de controle, devido à presença de autocorrelação, indicando instabilidade do processo no gráfico para a média. Quanto ao gráfico $\overline{M R}$, percebe-se que ele também está fora de controle estatístico.

Após tratar o efeito da autocorrelação presente nos dados por meio do modelo SARIMA $(2,1,0)(1,0,1)$, elaboram-se novamente os gráficos $\bar{x}$ e $\overline{M R}$, utilizando os resíduos do modelo selecionado, conforme as Figuras 12 e 13. Dessa vez, os efeitos de causas especiais poderão ser visualizados, e não mais os alarmes falsos.

X: -167,66 (-167,66); Sigma: 1205,9 (1205,9); n: 1,

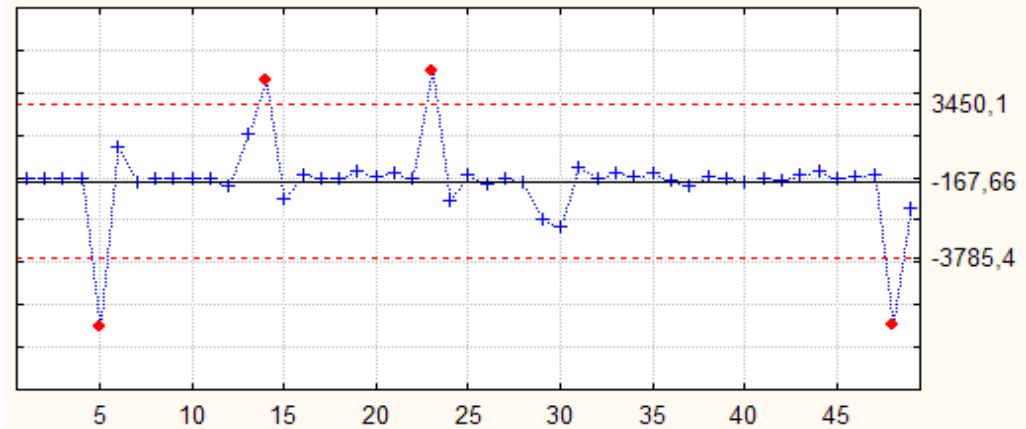

Figura 12. Gráfico $\bar{x}$ dos resíduos do modelo ARIMA.

MR: 1360,7 (1360,7); Sigma: 1028,0 (1028,0); n: 1,

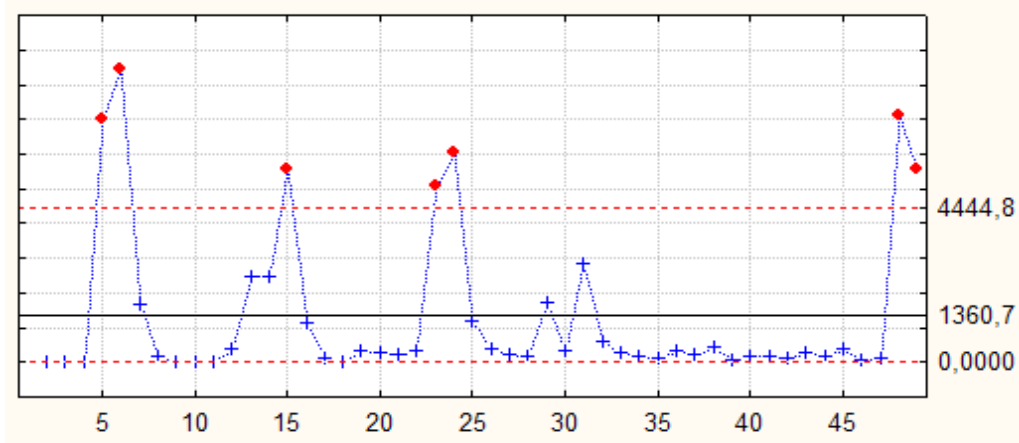

Figura 13. Gráfico $\overline{M R}$ dos resíduos do modelo ARIMA.

Analisando as Figuras 12 e 13, verifica-se que há poucos pontos amostrais fora dos limites de controle, se comparados aos gráficos $\bar{x}$ e 
$\overline{M R}$ da série original, apresentados nas Figuras 10 e 11. Isso indica que o modelo SARIMA foi capaz de captar as variações que havia na série produção de leite, pois o efeito da autocorrelação serial foi tratado.

Com relação aos resultados obtidos após a aplicação dos gráficos de controle $\bar{x}$, apresentam-se as seguintes considerações sobre os pontos amostrais fora de controle para a série produção de leite.

Nos dados originais da variável, verificou-se pelo gráfico $\bar{x}$ que há 9 pontos amostrais em sequência no mesmo lado da linha central, representados pelo conjunto de amostras iniciadas nas observações 6, 15, 24 e 33. Há, também, um conjunto de 7 amostras, em que, a cada 3 pontos amostrais traçados, 2 encontram-se localizados na zona A ou fora dela, correspondendo às observações com início nas amostras 5, 8, 11, 23, 26, 29 e 48. Igualmente, observa-se um conjunto de 9 amostras, em que, a cada5 pontos amostrais traçados, 4 encontram-se localizados na zona B ou fora dela, correspondendo às observações com início nas amostras 1 , $6,11,16,21,26,31,36$ e 41. Além disso, observam-se 8 pontos amostrais em sequência, situados fora da zona $\mathrm{C}$, correspondendo às observações com início em 1, 9, 17, 25, 33 e 41.

Por sua vez, ao analisar o gráfico $\bar{x}$ dos resíduos do modelo SA$\operatorname{RIMA}(2,1,0)(1,0,1)_{7}$, observaram-se15 pontos amostrais em sequência, situados na zona $\mathrm{C}$, os quais correspondem às observações do intervalo de 31 a 45 .

Mediante essas informações, constata-se que o modelo matemático foi adequado para tratar o problema da autocorrelação que havia na série. Assim, esse modelo evidenciou uma melhora significativa na detecção de pontos amostrais fora de controle estatístico.

Além disso, os gráficos de controle evidenciaram que a série produção de leite está fora de controle estatístico. De acordo como gráfico $\bar{x}$ dos resíduos do modelo apresentado na Figura 12, há 15 pontos amostrais em sequência situados na zona C. Isso é uma indicação de que a série está fora de controle. Portanto, os pontos amostrais fora de controle estatístico correspondem ao período de 22 de fevereiro a 31 de maio de 2010.

\section{Considerações finais}

Após a execução das etapas metodológicas, conclui-se que o procedimento proposto foi adequado. 
Aplicando os modelos ARIMA, a autocorrelação serial foi eliminada, indicando que os resíduos do modelo matemático encontrado apresentam-se aptos para uma análise futura por meio de gráficos de controle. Assim, pode-se afirmar que o modelo matemático SARIMA $(2,1,0)(1,0,1)_{7}$ captou bem o comportamento da série produção de leite semanal.

Com a série livre de autocorrelação, foram elaborados gráficos de controle nos dados originais e nos resíduos do modelo matemático. Ao comparar esses gráficos, constatou-se que os dados originais apresentaram uma maior quantidade de pontos amostrais fora dos limites de controle em relação aos resíduos do modelo matemático encontrado.

Nesse contexto, evidencia-se a importância dos modelos de previsão para as empresas que recolhem o leite fornecido pelos pequenos produtores do município de Santo Ângelo, como forma de fornecer informações quanto ao gerenciamento da produção nos períodos de menor e maior produção de leite.

Além disso, salienta-se que os produtores devem criar métodos alternativos para estocar o alimento para o gado leiteiro, investindo em rações alternativas e condições sanitárias, de modo que, no período de inverno, não ocorra uma redução tão significativa da produção de leite em relação aos períodos de maior produção.

6. Referências

BUENO, R. L. S. Econometria de séries temporais. São Paulo: Cengage Learning, 2008.

GUJARATI, D. N. Econometria básica. São Paulo: Makron Books, 2000. HILL, C.; GRIFFITHS, W.; JUDGE, G. Econometria. São Paulo: Saraiva, 1999.

KLIDZIO, R.; SOUZA. A. M. Modelos de previsão aplicados ao controle de qualidade com dados autocorrelacionados. Dissertação (Mestrado em Engenharia de Produção), Santa Maria: UFSM, 2009.

LÍRIO, G. W. et al. Análise e previsão da série recebimento e produção de leite da usina escola de laticínios da Universidade Federal de Santa Maria - RS. Revista Ciência \& Natura, v.25, p.23-39, 2003. 
MONTGOMERY, D. C. Introdução ao controle estatístico da qualidade. 4.ed. Rio de Janeiro: LTC, 2004.

MONTGOMERY, D. C. Introduction to statistical quality control. 3.ed. New York: John Wiley \& Sons Inc., 1997.

MONTGOMERY, D. C; RUNGER, G. C. Estatística aplicada e probabilidade para engenheiros. 2. ed. Rio de Janeiro: LTC, 2003.

MORETTIN, P. A. Econometria financeira: um curso em séries temporais financeiras. São Paulo:ABE, 2006.

RYAN, T.P. Statistical methods for quality improvement. New York: John Wiley \& Sons Inc., 1989.

WERNER, L.; RIBEIRO, J. L. D. Previsão de demanda: uma aplicação dos modelos Box Jenkins na área de assistência técnica de computadores pessoais. Revista Gestão \& Produção, v.10, n.1, p.47-67, abr. 2003.

SIQUEIRA, L. G. P. Controle estatístico do processo. São Paulo: Pioneira, 1997.

Submetido em: 02/01/2012

Aceito em: 14/12/2012 